\title{
Differentiation of fencing blade alloys using iPhone magnetometer
}

DOI: 10.30609/JETI.2020-9096

Lucas Braddock Chen ${ }^{1 *}$

1West Coast Fencing Academy, US Fencing, Monrovia, CA 91016, USA

\begin{abstract}
All modern fencing sabers are made of composite steel alloys with variable stiffness and tensile strength. Blade differentiation is often difficult due to similar weight, color, and appearance, yet blade quality is of utmost importance as Olympic fencers have died from broken blades. Traditional methods of steel alloy identification involve spectrometry or machine tensile strength assessment, which are impractical in a competition arena. Since all modern smartphones have built-in magnetometers to detect the Earth's magnetic field as a component of GPS and location services, we previously patented the utilization of the smartphone magnetometer to differentiate fencing steel alloys. This manuscript now reports the experimental protocol necessary for the novel application.
\end{abstract}

Keywords: Fencing, steel, alloy, iPhone, magnetometer, magnetism.

\section{Introduction}

The sport of fencing is as old as the history of warfare, as soldiers practiced and were drilled on sword fighting. Fencing became an official Olympic sport in the 1896 Athens Olympic Games[1], and all modern day fencing weapons are made with carbon tempered steel alloy[2].

Steel is primarily made of raw iron, which contains varying amounts of carbon $(0.25-2 \%)$, sulfur $(<0.05 \%)$, phosphorus $(<0.04 \%)$, and silicon $(0.3$ $0.5 \%$ [3]. Iron is then mixed with different elements, such as manganese, nickel, chromium, molybdenum, vanadium, silicon, boron, aluminum, cobalt, copper, cerium, niobium, titanium, tungsten, tin, zinc, lead, and zirconium, to give specific hardness, flexibility, and other properties [4]. 
Just like in the days of medieval warfare, the quality of a blade is important. A blade that has lower hardness is more easily corroded, deformed, and shattered. Despite the importance of blades, it is impossible for a fencer to differentiate between blades of different qualities as all blades have similar appearance and feel. Fencers rely upon brand labelling, but any brand, however famous or expensive, can have both good and inferior batches of steel.

The traditional methods of steel identification, such as appearance test (color and machine marks), spark test (grinding steel to generate sparks and to assess steel quality based on sparks color and quality), and the Rockwell or Brinell or Vickers tests (estimate steel hardness by measuring the depth or width of indentation from a pounding machine), are subjective and inaccurate. Modern tests are less subjective, but require expensive and cumbersome equipment, such as X-ray fluorescence and atomic emission spectrometry or laser-induced breakdown spectrometers[5]. None of these methods of steel identification are practical for a fencer in a sports arena, as they require heavy and specialized equipment. We now report a rapid method of steel identification based on their magnetic properties. Only a few elements have magnetic properties, including iron, nickel, cobalt, gadolinium, and dysprosium[6]. Since steel of different grades and quality contain different ratios of these elements, each blade has a different baseline magnetic profile (strength and direction of maximum magnetic field). In addition, since magnetic properties can change while conducting electricity, each blade will demonstrate unique magnetic profiles at different levels of electricity due to different electricity conductivity (dynamic magnetic property). By measuring the magnetic profile, a fencer can readily differentiate between different blades. This process is further facilitated by the prevalence of smartphones. All modern smartphones possess a magnetometer as a part of their internal compass[7], and we previously patented the utilization of these magnetometers to capture the blade's magnetic profile - both the static magnetic profile (before electric is applied) and the dynamic profiles (with different levels of electricity)[8].

The invention requires the following parts: test material, a test material holder, a smartphone with magnetometer, a stabilizing platform for the smartphone, an app for measuring electromagnetic profiles, an electric source, and wires to conduct electricity from the electric source to the test material. 


\section{Material and Methods}

i. Blades: all saber blades are purchased from either Absolute Fencing Gear, Inc (Bridgewater, NJ) or Allstar Fencing (US) Limited (Berkshire, United Kingdom). The blades were tested without the metal bell guard in order to minimize magnetic interference.

ii. Test Platform: blades were held by rubber insulators to preserve circuit integrity: Rubber Vise Clamp, Amazon (Seattle, WA).

iii. Smart phone: iPhones, XS Max, Apple (Cupertino, CA) running iOS 12.4 was used to run the magnetizer software.

iv. Phone Stabilizer: Aduro Solid-Grip Holder for Desk - Adjustable Universal Gooseneck Smartphone Stand by Entronik (Brooklyn, NY)

v. Software: Magnetscape 2.0 App by Toon, Llc (Osaka, Japan).

vi. Electric Source: 6 Volt Lantern Battery, Eveready (St. Louis, M0).

vii. Electricity wires: Alligator clip leads with 18 standard wire gauze stranded copper wires and vinyl sheath, RadioShack (Fort Worth, TX)

viii. Amp Meter: Multimeter vpro850L, WeePro by Amazon (Seattle, WA)

ix. Resistance: Resistance Substitution Box Model RS-400, Elenco Electronics (Wheeling, IL)

\section{Results and discussion}

Static Magnetic Profile: The smartphone is held by an insulating stabilizer to avoid magnetic interference, while the blade is held by two insulating platforms. Measurement is recorded by the Magnetscape app on the smartphone. Magnetic field strength and direction can be visualized (Figure 1)

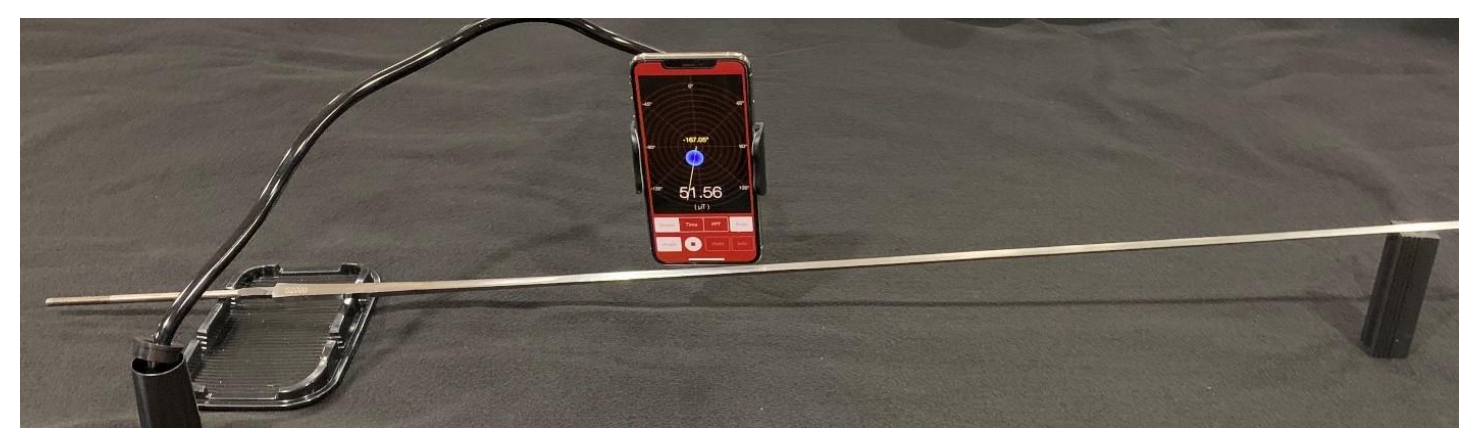

Figure 1: Baseline magnetic signatures were captured with the experimental platform as shown. 
Dynamic Magnetic Profile: A similar platform is used to record the various magnetic profiles at different current, with a variable resistor added in series (Figure 2)

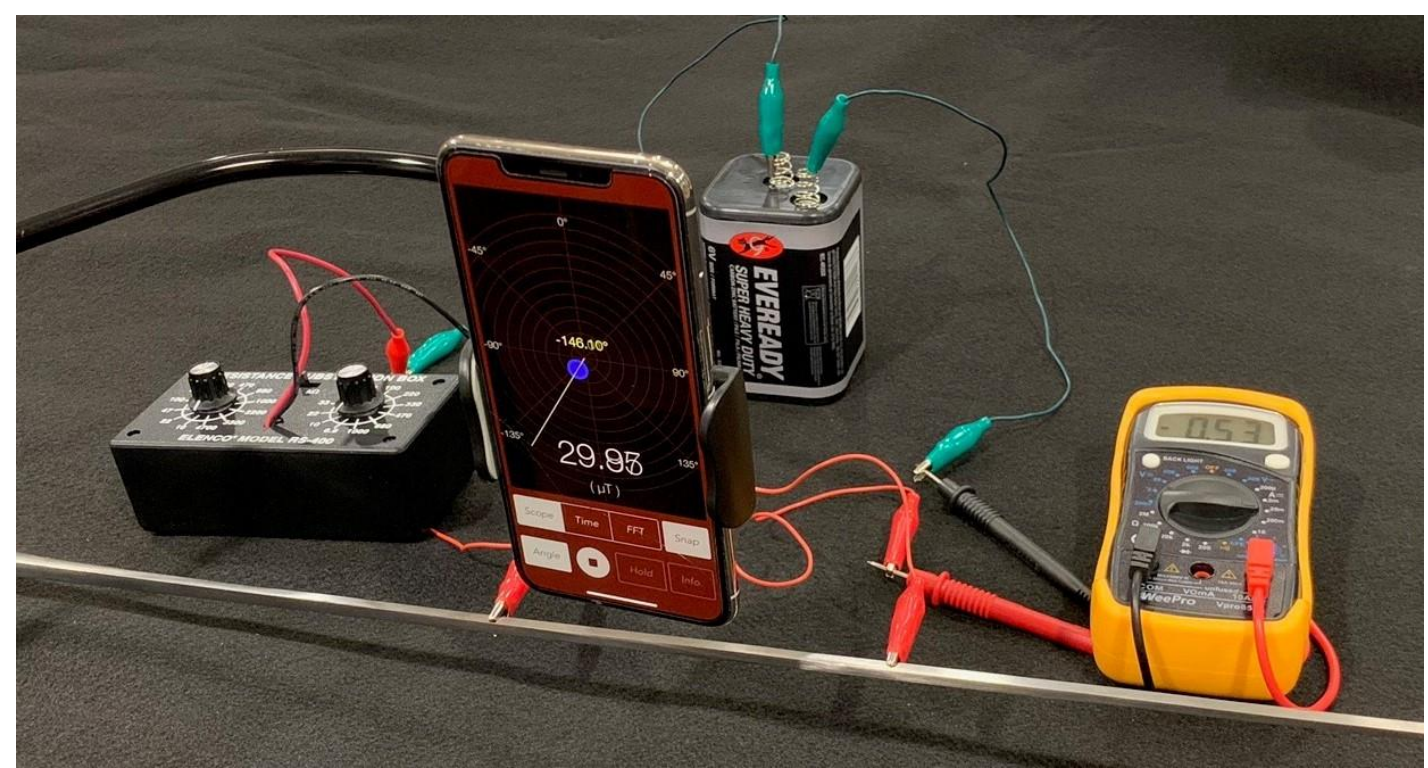

Figure 2: Dynamic magnetic signatures were captured once the blade was placed in the electromagnetic field.

The above experimental platform was able to capture the signatures from both the baseline as well as the dynamic setup. It is noted that not only do the magnetic signatures demonstrate a different value in the dynamic electromagnetic setting, but the vector of maximum magnetic force also varied in accordance to the electric charge.

\section{Conclusion}

The quality of a fencing blade is of upmost importance, both for the fairness of competition as well as for the safety of the athletes. It is difficult for a fencer to identify the quality of the blade due to their similar appearances and the absence of methods for field testing. We recently patented a method to differentiate steel alloys based on their unique magnetic profiles from different metal alloy compositions. We now describe the experimental protocol necessary to execute the method. It is expected that the method can be adopted by athletes to identify blades, and it may also provide a rapid method for referees and judges to verify and standardize the blades used in competition. 


\section{Bibliography}

[1] International Olympic Committee. Fencing: History of Fencing at the Olympics Games. Olympics Studies Centre. March 2015.

[2] AZOM. Advanced Materials in the Sport of Fencing. AZO Materials. March 15, 2001.

[3] Science Encyclopedia. Steel: Raw Materials. Downloaded September 18, 2019.

[4] Smith, William F, Hashemi, Javad. Foundations of Material Science and Engineering. McGraw-Hill, 2001. p. 394

[5] Vericheck Technical Services. Common Metal Detection Methods. December 1, 2016.

[6] Wallulis, Karl. List of Metals That Are Attracted to Magnets. April 17, 2018.

[7] TechBlog. Using techBASIC to Turn Your iPhone or iPad into a Metal Detector. November 30, 2011.

[8] Chen, Lucas. Determining Fencing Blade Quality using Dynamic Magnetic Field Measurements. The United States Trade and Patent Office, U.S. Serial Number 16/422,731. May 24, 2019. 